\title{
Sobre Finura Própria de Grafos
}

\author{
Moysés S. Sampaio Jr. ${ }^{1 *}$ Fabiano S. Oliveira ${ }^{2 \dagger}$ Jayme L. Szwarcfiter $^{1,2 \ddagger}$ \\ ${ }^{1}$ COPPE/PESC - Universidade Federal do Rio de Janeiro (UFRJ), Brasil \\ ${ }^{2}$ IME - Universidade do Estado do Rio de Janeiro (UERJ), Brasil \\ moysessjecos.ufrj.br, fabiano.oliveira@ime.uerj.br, jayme@nce.ufrj.br
}

\begin{abstract}
Both graph classes of $k$-thin and proper $k$-thin graphs have recently been introduced generalizing interval and unit interval graphs, respectively. The complexity of the recognition of $k$-thin and proper $k$-thin are open, even for fixed $k \geq 2$. In this work, we introduce a subclass of the proper 2-thin graphs, called proper 2-thin of precedence. For this class, we present a characterization and an efficient recognition algorithm.
\end{abstract}

\section{Introdução}

A classe dos grafos $k$-finos foi introduzida em [Mannino et al. 2007] como uma generalização da classe dos grafos de intervalo. Motivados por isto, [Bonomo, Estrada 2017] definiram a classe dos grafos $k$-finos próprios que, de forma similar, generalizam os grafos de intervalo próprio. As complexidades dos problemas de reconhecer os grafos $k$-finos e $k$-finos próprios, mesmo para $k \geq 2$ fixo, encontram-se em aberto. Neste trabalho, estudamos uma subclasse dos grafos $k$-finos próprios, que chamamos de $k$-finos próprios de precedência, para a qual apresentamos uma caracterização estrutural e um algoritmo eficiente de reconhecimento.

Um grafo de intervalo $G$ é um grafo tal que $V(G)$ é uma família de intervalos fechados da reta real, chamado de modelo, tal que $(I, J) \in E(G)$ se, e somente se, $I \cap J \neq \emptyset$. Em [Olariu 1991], mostra-se que um grafo $G$ é de intervalo se, e somente se, existir uma ordenação $<\operatorname{de} V(G)$ tal que, para qualquer tripla ordenada $(p, q, r)$ de vértices de $G$, se $(p, r) \in E(G)$, então $(q, r) \in E(G)$. Tal ordenação < é chamada canônica de $V(G)$. A Figura 1(a) ilustra um grafo de intervalo e uma de suas ordens canônicas. Um grafo de intervalo $G$ é dito próprio se admite um modelo tal que $I \nsubseteq J$, para todo $I, J \in V(G)$ distintos. Similarmente, $G$ é um grafo de intervalo próprio se, e somente se, admite uma ordenação $<$ de $V(G)$ tal que, para qualquer tripla ordenada $(p, q, r)$ de vértices, se $(p, r) \in E(G)$ então $(p, q),(q, r) \in E(G)$ [Roberts 1969]. Tal ordenação é denominada de canônica própria, como ilustrado na Figura 1(b).

Um grafo $G$ é denominado $k$-fino se existir um $k$-particionamento de $V(G)$ e uma ordenação associada (chamada de consistente) tal que, para qualquer tripla ordenada $(p, q, r)$ de $V(G)$, se $p$ e $q$ pertencerem a uma mesma parte e $(p, r) \in E(G)$, então $(q, r) \in E(G)$. Um grafo $G$ é dito $k$-fino próprio se existir uma $k$-partição de $V(G)$ e uma ordenação associada (chamada de fortemente consistente) que é consistente e tal que, para qualquer tripla ordenada $(p, q, r)$ de $V(G)$ se $q$ e $r$ pertencerem a uma mesma

\footnotetext{
${ }^{*}$ financiado por CAPES.

${ }^{\dagger}$ parcialmente financiado por FAPERJ.

${ }^{\ddagger}$ parcialmente financiado por CNPq.
} 


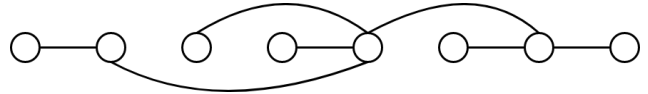

(a)

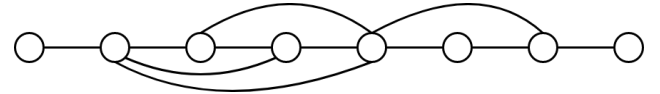

(b)

Figura 1. Exemplos de sequências (a) canônicas e (b) canônicas próprias.

parte e $(p, r) \in E(G)$, então $(p, q) \in E(G)$. O valor mínimo de $k$ para o qual $G$ é $k$-fino (resp. $k$-fino próprio) é chamado de finura (resp. finura própria) de $G$, denotado por thin $(G)$ (resp. pthin $(G)$ ). Note que, os grafos $k$-finos são uma generalização dos grafos de intervalo, e que os grafos $k$-finos próprios generalizam os grafos de intervalo próprios.

Em [Bonomo, Estrada 2017], é apresentado um algoritmo eficiente para determinar o particionamento mínimo de $V(G)$ para o qual uma dada ordenação é consistente, ou fortemente consistente. Além disso, prova-se que o problema de determinar se existe uma ordenação consistente, ou fortemente consistente, dado um particionamento é NPcompleto. De uma maneira geral, mesmo para $k \geq 2$ fixo, a complexidade de decidir se um grafo é $k$-fino ou $k$-fino próprio encontra-se em aberto. Neste trabalho investigamos o problema para uma classe de grafo mais restrita, definida por admitir uma ordenação fortemente consistente que goza de uma propriedade especial. Mais especificamente, chamaremos um grafo $G$ de 2-fino próprio de precedência (2-FPP) se $G$ é um grafo 2-fino próprio que admite bipartição $(X, Y)$ de $V(G)$ e uma ordenação fortemente consistente $<$ na qual todos os vértices de $X$ precedem aqueles de $Y$ em $<$. A Figura 2(a) ilustra um grafo que é 2-FPP e, na Figura 2(c), é ilustrado um grafo que não é 2-FPP com respeito a bipartição escolhida. A convenção da representação destas figuras é a de que os vértices das partes da bipartição $(X, Y)$ associada são dispostos horizontalmente, cada parte em uma horizontal, e a ordem da esquerda para direita é aquela encontrada em uma ordenação fortemente consistente. Além disso, por convenção, os vértices que estão embaixo precedem os que estão em cima na ordenação fortemente consistente.

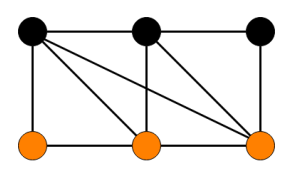

(a)

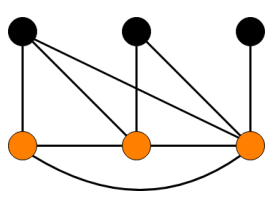

(b)

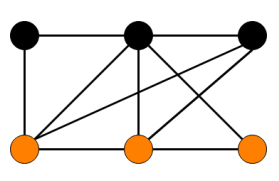

(c)

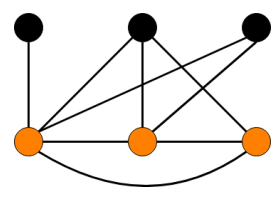

(d)

Figura 2. (a)(c) Grafos e bipartição $(X, Y)$ dados; (b)(d) Grafos $S(X, Y)$.

\section{Reconhecimento dos Grafos 2-FPP com Dada Bipartição}

Nesta seção, apresentaremos um algoritmo eficiente para o seguinte problema:

\begin{tabular}{ll}
\hline Problema: & Reconhecimento dos grafos 2-FPP com bipartição dada \\
Entrada: & Um grafo $G$ e uma bipartição $(X, Y)$ de $V(G)$ com $G[X]$ e $G[Y]$ conexos \\
QueStão: & $\begin{array}{l}\text { Existe uma ordenação }<\text { de } V(G) \text { consistente com }(X, Y) \text { tal que } \\
\text { cada vértice de } X \text { precede cada vértice de } Y \text { em }<\text { ? }\end{array}$ \\
\hline
\end{tabular}

Naturalmente, $G[X]$ e $G[Y]$ devem ser grafos de intervalo próprio. Em caso negativo, o algoritmo pode responder NÃO. Em caso positivo, sabe-se que $G[X]$ admite uma única ordenação canônica a menos de reversão e permutação entre os vértices gêmeos (vértices 
com mesma vizinhança fechada em $G[X]$ ). O mesmo pode ser dito para $G[Y]$. Sejam $s_{X}$ e $s_{Y}$ ordenações canônicas arbitrárias de $G[X]$ e $G[Y]$, respectivamente. Denote por $s^{-1}$ a reversão de uma ordenação $s$. O algoritmo prossegue para cada $\left(s_{1}, s_{2}\right) \in\left(\left\{s_{X}, s_{X}^{-1}\right\} \times\right.$ $\left.\left\{s_{Y}, s_{Y}^{-1}\right\}\right)$ verificando se existe uma ordenação $s$, obtida da concatenação de $s_{1}$ com $s_{2}$ e de eventual permutação de vértices que são gêmeos em $G[X]$ ou em $G[Y]$, que seja fortemente consistente com $(X, Y)$. Tal verificação é feita da seguinte forma.

O algoritmo utilizará um digrafo $D_{G}$ associado a $G$ no qual as arestas direcionadas representam ordens forçadas entre os vértices de $s$. Caso hajam relações de ordem mutuamente contraditórias, o que consiste na aparição de um ciclo em $D_{G}$, o par $\left(s_{1}, s_{2}\right)$ vigente é ignorado e o próximo é considerado. Caso contrário, qualquer ordenação que respeite as relações definidas pelas arestas de $D_{G}$ será consistente com $(X, Y)$, situação que o algoritmo responderá SIM e retornará uma ordenação topológica de $D_{G}$ como um certificado dessa resposta. Se um ciclo for encontrado em cada par $\left(s_{1}, s_{2}\right)$ investigado, o algoritmo responderá NÃO e retornará como certificado da resposta negativa os ciclos encontrados. As regras que forçam ordens relativas entre vértices serão as seguintes:

(i) Seja $X^{\prime}$ (resp. $Y^{\prime}$ ) o conjunto formado pelo último vértice de $s_{1}$ e seus gêmeos em $G[X]$ (resp. o conjunto formado pelo primeiro vértice de $s_{2}$ e seus gêmeos em $G[Y])$. Para todo $u \in X^{\prime}, v \in Y^{\prime},(u, v)$ é uma aresta forçada em $D_{G}$;

(ii) para todo $u, v \in X$, se $u$ e $v$ não são gêmeos em $G[X]$ e $u<v$ em $s_{1}$, então $(u, v)$ é uma aresta forçada em $D_{G}$. Analogamente, para todo $u, v \in Y$, se $u$ e $v$ não são gêmeos em $G[Y]$ e $u<v$ em $s_{2}$, então $(u, v)$ é uma aresta forçada em $D_{G}$;

(iii) para todo $u, v \in X$ e $w \in Y$, se $(u, w) \notin E(G)$ e $(v, w) \in E(G)$, então $(u, v)$ é uma aresta forçada em $D_{G}$. Analogamente, para todo $u, v \in Y$ e $w \in X$, se $(u, w) \in E(G)$ e $(v, w) \notin E(G)$, então $(u, v)$ é uma aresta forçada em $D_{G}$.

$\mathrm{O}$ algoritmo pode ser implementado em tempo $O\left(n^{2}\right)$.
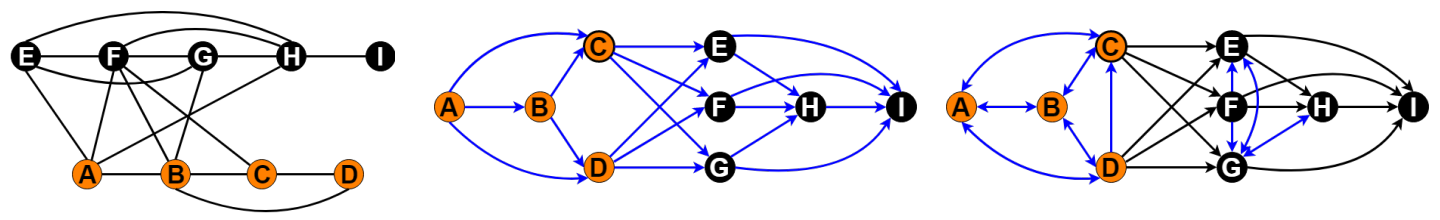

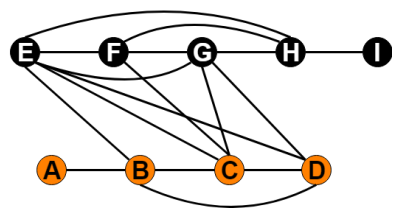

(a)

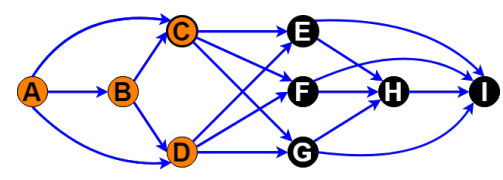

(b)

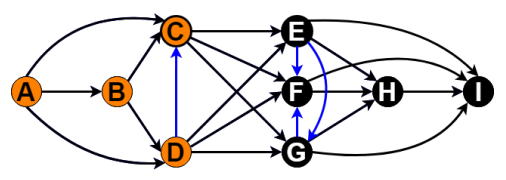

(c)

Figura 3. Dois exemplos de execução do algoritmo. (a) grafo e bipartição de entrada; (b) $D_{G}$ considerando arestas forçadas nos passos (i) e (ii); (c) $D_{G}$ considerando arestas forçadas no passo (iii).

\section{Caracterização dos Grafos 2-FPP com Dada Bipartição}

Nesta seção apresentamos uma caracterização dos grafos 2-FPP com dada bipartição. Um grafo $G$ é um grafo de divisão se existir uma bipartição $(X, Y)$ de $V(G)$ tal que $X$ seja uma clique e $Y$ um conjunto independente. Um grafo é dito de limiar se $G$ é um grafo de 
divisão que admite uma ordenação dos vértices de $X$ (resp. $Y$ ), chamada de ordenação de limiar, de modo que suas vizinhanças estejam ordenadas por inclusão, isto é, se $u$ precede $v$ na ordenação, então $N[u] \subseteq N[v]$ (resp. $N(u) \subseteq N(v)$ ). Para a caracterização a seguir, definiremos o grafo de divisão $S(X, Y)$ associado à bipartição de entrada $(X, Y)$, obtido de $G$ pela adição e remoção de arestas de modo que $X$ se torne uma clique e $Y$ se torne um conjunto independente. As Figuras 2(b) e 2(d) ilustram os grafos de divisão associados aos grafos das Figuras 2(a) e 2(c), respectivamente.

Teorema 1. Se Gé um grafo e $(X, Y)$ é uma bipartição de $V(G)$, então $G$ é 2-FPP com respeito a $(X, Y)$ se, e somente se, existir ordenações canônicas $s_{X}, s_{Y}$ de respectivamente $G[X]$ e $G[Y]$ tais que $S(X, Y)$ é um grafo de limiar para o qual $s_{X}$ corresponda a uma ordenação de limiar de $X$ e $s_{Y}^{-1}$ a uma ordenação de limiar de $Y$.

Demonstração. Suponha que $G$ é 2-FPP com respeito a bipartição $(X, Y)$ de $V(G)$ e $s$ é uma ordenação consistente própria associada. Seja $s_{X}$ (resp. $s_{Y}$ ) o prefixo (resp. sufixo) de $s$ correspondente aos vértices de $X$ (resp. $Y$ ). Naturalmente, $s_{X}$ e $s_{Y}$ são ordens canônicas de $G[X]$ e $G[Y]$, respectivamente. Suponha que $s_{X}$ não é uma ordenação de limiar de $X$. Então, existem $u, v \in X$ e $w \in Y \operatorname{com} u<v$ em $s_{X}$ tais que $w \in N[u]$ e $w \notin N[v]$. Mas então $(u, w) \in E(G),(v, w) \notin E(G)$ e $u<v<w$ em $s$, contrariando $s$ ser uma ordenação fortemente consistente. Suponha agora que $s_{Y}^{-1}$ não é uma ordenação de limiar para $Y$, e logo existem $u, v \in Y$ e $w \in X \operatorname{com} u<v$ em $s_{Y}$ tais que $w \in N(v)$ e $w \notin N(u)$. Mas então $(v, w) \in E(G),(u, w) \notin E(G)$ e $w<u<v$ em $s$, o que contraria $s$ ser uma ordenação fortemente consistente. Por outro lado, considere que $s_{X}$ e $s_{Y}^{-1}$ são ordenações de limiar de $X$ e $Y$ e canônicas de $G[X]$ e $G[Y]$. Mostraremos que $s=s_{X} s_{Y}$ é uma ordenação fortemente consistente com respeito a bipartição dada $(X, Y)$, seguindo o resultado. Na suposição do contrário, existiria (i) $u, v \in X, w \in Y \operatorname{com} u<v$ em $s$ tais que $(u, w) \in E(G),(v, w) \notin E(G)$, ou (ii) $u, v \in Y, w \in X$ com $u<v$ em $s$ tais que $(v, w) \in E(G),(u, w) \notin E(G)$. No caso (i) (resp. (ii)), há contradição com o fato de $s_{X}$ (resp. $\left.s_{Y}^{-1}\right)$ ser uma ordenação de limiar para $X$ (resp. $Y$ ).

\section{Conclusão}

Este trabalho foi motivado pelos problemas de determinar a finura, e a finura própria em grafos. A introdução de ambos os parâmetros é um conceito relativamente recente e foi motivada por uma generalização do bem-conhecido problema de reconhecimento de grafos de intervalo e de intervalo unitários. A complexidade de reconhecer se um grafo é $k$-fino ou $k$-fino próprio encontra-se em aberto mesmo para $k \geq 2$ fixo. Neste trabalho, introduzimos a classe dos grafos 2-finos próprios de precedência (2-FPP) e apresentamos um algoritmo de reconhecimento polinomial e uma caracterização estrutural da classe.

\section{Referências}

Bonomo, F., Estrada, D. (2017). On the thinness and proper thinness of a graph. CoRR, abs/1704.00379.

Mannino, C., Oriolo, G., Ricci, F.,, Chandran, S. (2007). The stable set problem and the thinness of a graph. Operations Research Letters, 35:1-9.

Olariu, S. (1991). An optimal greedy heuristic to color interval graphs. Information Processing Letters, 37:21-25.

Roberts, F. (1969). Indifference graphs. Em Harary, F., editor, Proof Techniques in Graph Theory, p. 139-146. Academic Press, New York. 